\title{
The Impact of Eruptions from Young Stars on Environments of Rocky Exoplanets
}

\author{
Vladimir S. Airapetian ${ }^{1,2}$ \\ ${ }^{1}$ NASA Goddard Space Flight Center, Greenbelt, MD USA \\ ${ }^{2}$ American University, Washington, DC, USA
}

\begin{abstract}
Kepler and TESS missions have discovered over 4500 extra solar (exoplanets) around F, G, K and M dwarfs. They also revealed frequent superflares on planet hosting stars, providing a mechanism by which host stars may have profound effects on the physical and chemical evolution of exoplanetary atmospheres. While we can only infer the course of the Sun's early evolution and how it might have affected the early evolution of the Earth, possibly setting the stage for the origin of life, the observation of planets around sun-like stars allows us to directly observe events which likely took place in our own solar system. A major question this leads to is: what effects do extreme energy fluxes from eruptive events during evolution of G-K planet hosts have on prebiotic chemistry and primitive life forms on primitive planets? To address this question, I will describe recent observations of young solar-like stars as inputs for our 3D MHD models of the corona, the wind and transient events (flares, coronal mass ejections and solar energetic particle events) and discuss their impact on atmospheric erosion and chemistry of our planet. I will then use these constrained energy fluxes to describe our recent atmospheric chemistry models impacted by energetic particles from the young Sun and formation and precipitation of biologically relevant molecules. I will then highlight our results of laboratory experiments of proton irradiation of mildly reduced gas mixtures and their implications to the climate, prebiotic chemistry and the rise of habitability on early Earth and young exoplanets.
\end{abstract}

Keywords: Solar-like Stars, Coronae, Winds, Coronal Mass Ejections, Stellar Energetic Particle Events, Exoplanets, Atmospheric Chemistry

\section{Introduction}

The search for habitable planets and signatures of extraterrestrial life is one of the most ambitious and consequential endeavors of our civilization. Explosion of Kepler and current Transiting Exoplanets Survey Satellite (TESS) led detection of Earth-sized exoplanets within habitable zones opened a new avenue in physical characterization of their atmospheres with the goal to search for the signatures of life. Current data suggest that rocky exoplanets should be common around G-M dwarfs (Zink et al. 2019). Detection of rocky exoplanets within habitable zones around active planet hosting stars suggests that many rocky exoplanets can be exposed to large fluxes of ionizing radiation from coronae and flare activity of planet hosts. This raises the science question we will explore in this project: What is the impact of stellar ionizing radiation fluxes on habitability of rocky worlds around active G-K stars? Our team has recently presented the roadmap for the 31 study of various aspects of star-planet interactions in a global exoplanetary system environment with a systematic, integrated approach using theoretical modeling, observational and laboratory methods combining tools and methodologies of astrophysics, heliophysics, planetary and Earth science as shown in Figure 1 (Airapetian et al. 2020).

To address this question, we need to reconstruct the properties of eruptive events from active G-K dwarfs. Unlike the Sun and solar-type (G-type) stars, $\mathrm{K}$ dwarfs can retain their magnetic activity for billions of years (Loyd et al. 2016). Most of young solar-like ( $\mathrm{G}$ and K-type) stars show signatures of powerful flares with energy exceeding the largest solar flares by a factor of i 10 (referred to as superflares; Airapetian et al. 2020). Flare driven X-ray and Extreme UV (referred to as XUV, 1-120 nm), CME driven shocks and associated SEP fluxes can have a crucial impact on atmospheric escape 22 and chemistry, and thus on climate evolution and habitability of young exoplanets around active planet hosts (Cohen et al. 2014; Airapetian et al. 2016; 2017a; 2017b; 2020; Airapetian 2017; Garcia-Sage et al. 2017; Dong et al. 2018). 
Recent Kepler, TESS and XMM-Newton, CHANDRA and NICER observations suggest that young solar-like ( $\mathrm{G}$ and $\mathrm{K}$ type) stars show large starspots covering up to $10 \%$ of a stellar surface, strong surface magnetic fields up to a few hundred Gauss, The strong surface magnetic flux drives dense hot X-ray bright coronae and massive fast winds, while coronal active regions supply energy for energetic flares (Sanz-Forcada et al. 2011; 2019; Gudel 2007; Maehara et al. 2012; Kochukhov et al. 2020; Notsu et al. 2019; Airapetian et al. 2020).

While these processes can be important for evolution of stellar angular momentum and their contribution to magnetic breaking and activity, explosive events can impact environments of rocky exoplanets in the habitable zones around these stars and present challenges for their habitability (see Dong et al. 2018; Yamashiki et al. 2019; Airapetian et al. 2017; 2020 in references herein). Specifically, ionizing radiation fluxes in the X-ray [0.1-10 nm] and Extreme UV (EUV, 10-91.2 nm) bands (referred to as the XUV band) can deposit heating via photoinization in the exospheres of exoplanets, and thus cause atmospheric escape (Cohen et al. 2014; Johnstone et al. 2019; Airapetian et al. 2020; Vidotto \& Cleary 2020). The moderate ionizing fluxes (about 10 times of the current Sun's EUV flux) can also erode planetary atmospheres via production of photoelectrons that drive polarization electric field, the source of ion outflows from exoplanets (Airapetian et al.2017; Garcia-Sage et al. 2017). The recent model of Johnstone et al. 2019 suggest that as stellar XUV fluxes greater than 60 times of the current Sun significantly increases neutral temperature with overall exospheric expansion transitioning to the hydrodynamic escape. Massive and magnetized stellar winds from young stars can exert dynamic pressure on exoplanetary magnetospheres, and thus leading to the expansion of the the polar cap area as well as generate ionospheric currents that can dissipate their energy via Joule heating, which is another factor of atmospheric escape via thermal plasma expansion (Cohen et. al. 2014; 2018). Thus, accurate knowledge of the XUV and wind fluxes are critical factors in assessing the atmospheric escape rates, and thus atmospheric evolution of Venus, Earth and Mars and exoplanets.

The XUV fluxes are enhanced by a factor of 10-100 during large stellar flares, and thus exoplanetary atmospheres around young stars irradiated with frequent flare events can be subject to high escape fluxes and rapid loss of atmospheres. However, coronal mass ejections associated with stellar flares can drive formation of particles up to a few $\mathrm{GeV}$ in their shocks that can modify the atmospheric chemistry of exoplanets via formation of biologically relevant molecules that can precipitste to the exoplanetary surfaces. Here, we provide a brief review of our recent results in modeling coronal stellar environments and their impact on chemistry of exoplanets.

\section{Coronae and Winds of Active Solar-like Stars}

Coordinated multi-wavelength multi-observatory efforts based on HST, XMM-Newton, Chandra, NICER, and ground-based photometric, spectropolarimetric observations are a crucial program for the characterization of input magnetic field and energy fluxes from G-M dwarfs required for their theoretical modeling. This is because stellar surface magnetic field and Zeeman Doppler Images based on spectropolarimetric observations of young $\mathrm{G}, \mathrm{K}$ and $\mathrm{M}$ dwarf stars provide information about large scale structures of the surface magnetic field. They reveal magnetic field strengths much greater than that observed on the Sun (e.g. Rósen et al. 2016; See et al. 2019). The photospheric magnetic field strengths span the range between 10 G to several $100 \mathrm{G}$ in young Sun-like stars, and up to a few $1000 \mathrm{G}$ for M-dwarfs (Kochukhov et al. 2021). They also shows a great diversity of geometry of large-scale stellar magnetic fields varying from poloidal to toroidal configurations (Vidotto et al. 2014). Recent observations revealed that the geometry of global magnetic fields and associated Poynting fluxes may vary on time scales of a few days to years such as BE Cet, HN Peg, eps Eri, $\kappa^{1}$ Cet, EK Dra, AU Mic, Proxima Cen (Boro Saikia et al. 2015; Rosen et al. 2016; Waite et al. 2017). The total unsigned magnetic flux accounts for over $90 \%$ of the magnetic flux and is mostly concentrated in stellar starspots (Kochukhov et al. 2020).

It is generally understood that the magnetic field is the ultimate source of energy for coronal heating and wind acceleration, the details of how magnetic energy is transferred from the solar photosphere into the corona remain poorly known. One promising heating mechanism involves the generation and propagation of the Alfvénor magnetosonic waves driven by photospheric motions depositing their energy into the chromosphere, transition region and corona via resonant absorption, phase mixing, or turbulent dissipation (). Solar observations provide evidence for energy transport via upward-propagating large-amplitude Alfvénwaves in the solar chromosphere, transition region, and corona, where the wave amplitudes are inferred from the Doppler line-of-sight velocity perturbation and non-thermal broadening of optically thin 
emission lines (Cranmer \& Winebarger 2019)

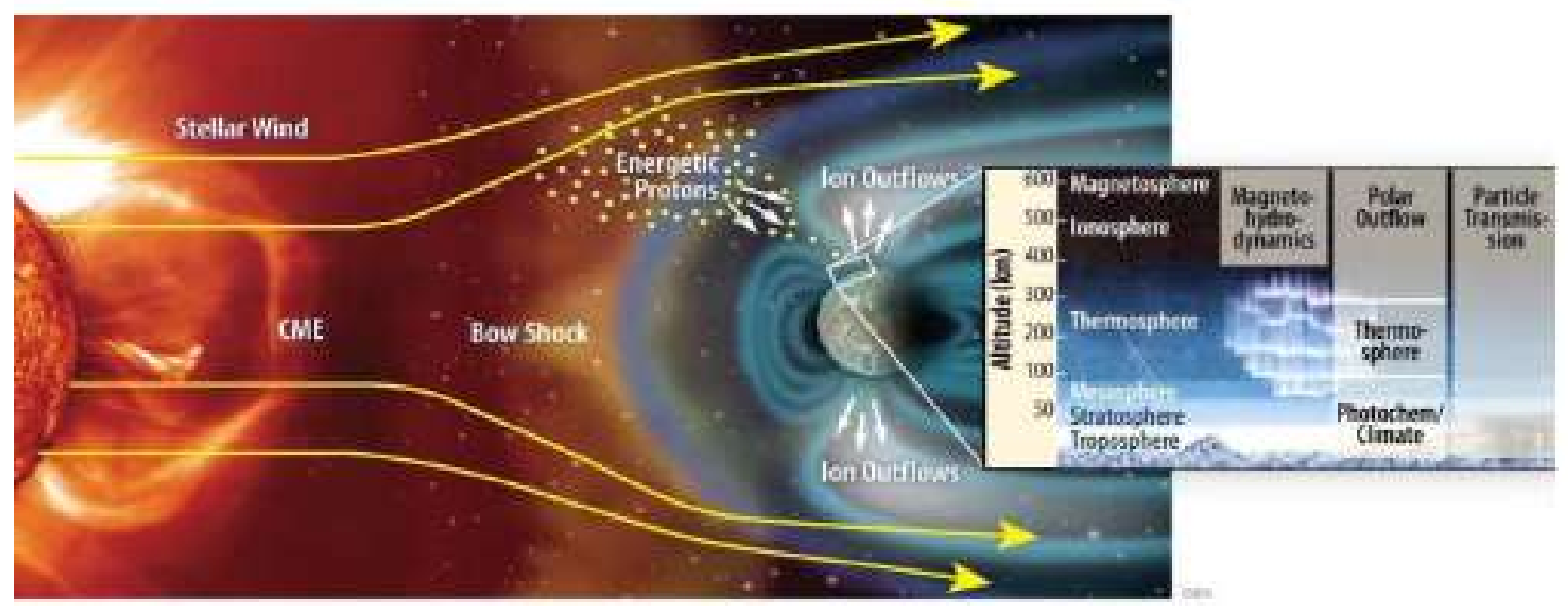

Figure 1. Schematic view of the complex exoplanetary space weather system that incorporates the physical processes driving stellar flares, coronal mass ejections (CMEs), their interactions with an exoplanetary atmosphere. While stellar winds and CMEs affect the shape of an exoplanetary magnetosphere, X-ray and Extreme Ultraviolet Emission (XUV) and stellar energetic particles (SEP) accelerated by coronal flares or CME-driven shocks enter the atmosphere.

Recently, Alvarado-Gomez et al. 2016; 2018; De Nascimento et al. 2016 Boro Saikia et al. (2020) used AlfvénWave Solam Model (AWSoM) constrained with ZDI magnetograms of active stars as boundary for realistic three-dimensional magnetohydrodynamic models of the coronae and winds of active solar-like stars. Airapetian et al. (2021) have extended these models by using fully resolved profiles of chromospheric lines from the Hubble Space Telescope observations that constrain the energy flux of Alfvénwaves at the upper chromosphere that propagate upward and dissipate energy in the upper layers forming the stellar corona.
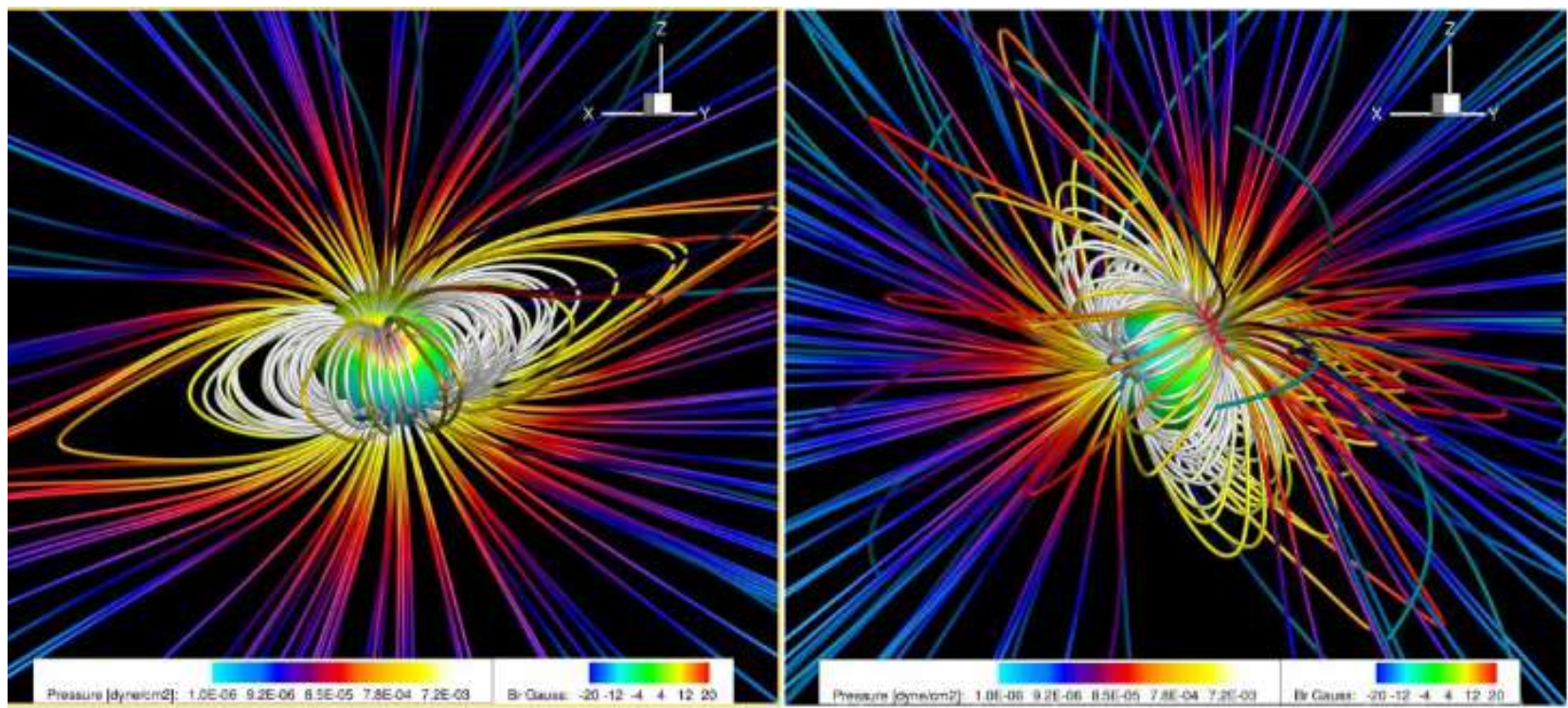

Figure 2. Global structure of the stellar magnetic corona of $\kappa^{1}$ Ceti at 2012.9 (left panel) and 2013.8 (right panel) with the superimposed plasma pressure along the field lines specified by the color bars.

The coronal models of $\kappa^{1}$ Ceti developed by Airapetian et al. (2021) is presented in Figure 2. The figure shows that the converged (steady) solution for the global magnetic coronal structure of the star at 2012.8 epoch, which is mostly represented by a dominant dipolar field tilted at 90 (dipole strength of $15.38 \mathrm{G}$ vs 5.1 $\mathrm{G}$ and 7.99 G for quadrupole and octupole respectively at 2013.7) and resembles the current Sun's coronal state at minimum of the solar cycle. The right panel demonstrates the magnetic filed topology 11 months later suggesting that the stellar dipole magnetic field has undergone a dramatic transition from a simple 
dipole to 450 tilted dipolar magnetic field with over $2 / 3$ of the contribution from quadrupolar and octupal components).

The MHD model also provides steady wind solutions for 2012.8 and 2013.7 epochs. The global wind shows the two-component stellar wind of $\kappa^{1}$ Ceti. The plasma accelerated from giant coronal holes (see Figure 4) is driven by the Alfvénwave ponderomotive force, while slow wind is formed due to the thermal pressure gradient. At 2012.8 epoch, the fast wind reaches its terminal velocity of $1152 \mathrm{~km} / \mathrm{s}$ within the first $15 R_{\text {star }}$, while the slow and dense component of the wind originates from the regions associated with the equatorial streamer belt structures at $696 \mathrm{~km} / \mathrm{s}$.

The simulated stellar wind density is $\sim 50-100$ times greater than that of the current Sun's wind and faster by a factor of 2 , which produces a massive wind with the mass loss rates of $2.8 \times 10^{-12} M_{\text {sun }}$, which is 100 times greater than that observed from the current Sun. As high-speed solar wind originating in the stellar coronal hole structures interacts with the preceding slower wind formed along the equatorial regions of the stellar corona, it forms a region of compressed plasma, SIR, along the leading edge of the stream, which, due to the rotation of the star at 9.2 days, is twisted approximately into an Archimedean (or Parker, 1958) spiral. Because the coronal holes may persist for many months, the interaction regions and high-speed streams tend to sweep past an exoplanet at regular intervals of approximately the half of the stellar rotation period forming Corrotating Interaction Regions (CIRs) along the Parker spirals.
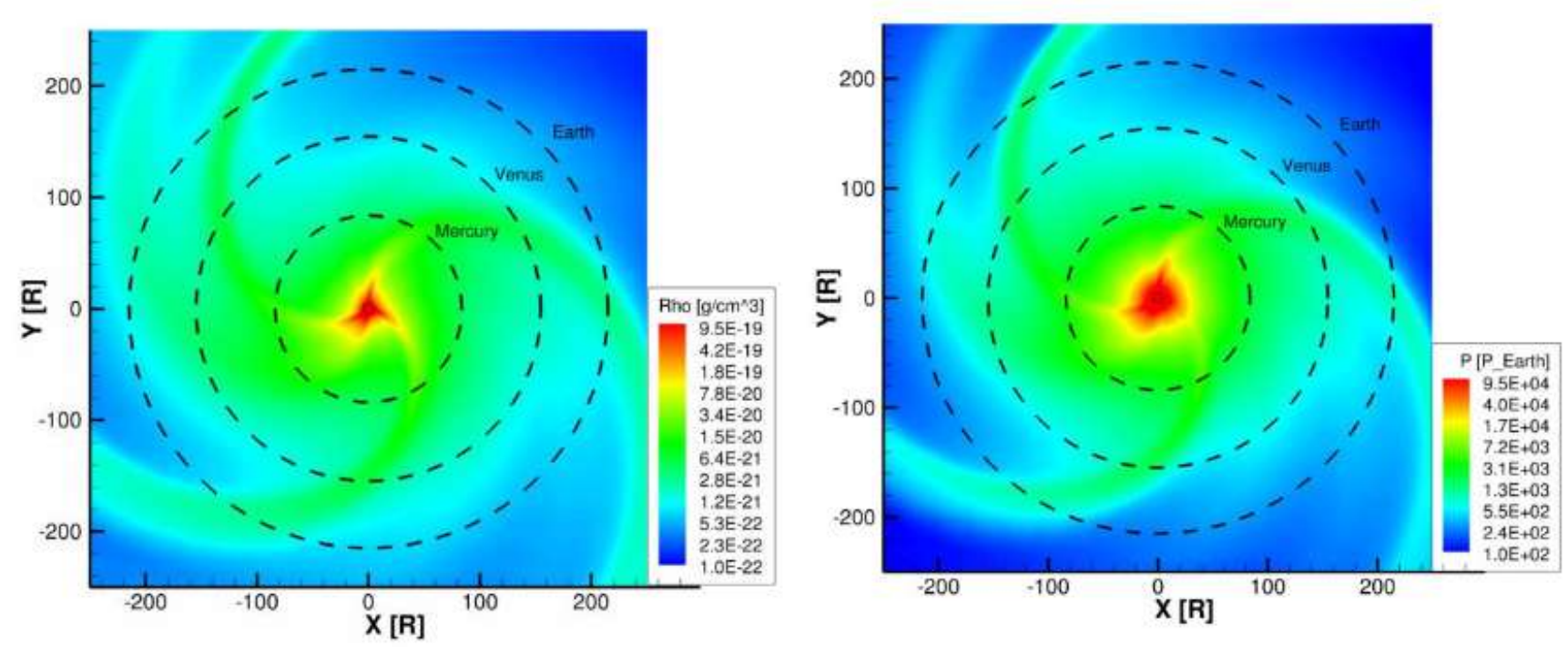

Figure 3. The top panel: $2 \mathrm{D}$ slice of the $\mathrm{X}-\mathrm{Y}$ plane $(\mathrm{Z}=0)$ of the plasma density and the dynamic pressure of CIRs from $\kappa_{1}$ Ceti formed at 2012.8 epoch. Black dashed lines show the orbits of Mercury, Venus and Earth respectively.

The CIR wind densities are about 300 greater than the solar wind's density measured by Parker Solar Probe. These results can be applied to characterization of physical conditions at orbital distances of close-in exoplanets around G and K -types stars including eps Eri b (Benedict et al. 2016), K2-229b (Santerne et al. 2018), K2-198b.c, K2-168b,c (Hedges et al. 2016), HD 189733b (Barth et al. 2021). The simulated dynamic pressure exerted by CIRs at close-in exoplanets reaches 5,000-10,000 times at 0.05 AU and 1300 times greater at $1 \mathrm{AU}$ than that exerted to the magnetosphere of the current Earth. The dynamic pressure from stellar CIRs would compress the magnetospheres of Earth-like exoplanets (at the current Earth's magnetic moment) to the standoff distance of $\sim 3 R_{\text {Earth }}$, and thus ignite strong geomagnetic currents in the early Earth atmosphere.

\section{Superflares, Coronal Mass Ejections and Stellar Energetic Particle Events}

The evolution of stellar magnetic field has dramatic effects on the frequency and energy of stellar superflares from active stars driven by the rapid release of free magnetic energy stored in the sheared and/or twisted strong fields typically associated with sunspots and active regions (e.g. Shibata \& Magara 2011; Maehara et al. 2020; Kazachenko et al. 2012). The maximum energy of superflares from young G-K 
stars decreases with age from from 1036 ergs (young stars with periods of 1-3 days) to $10^{34}$ ergs ( $>20$ days). Because large (white-light) solar flares are accompanied by CMEs with the energy comparable (or exceeding) to solar flares, we can expect that stellar superflares from young solar-like stars should also be associated with energetic CMEs with energies up to 1036 ergs (Gopalswamy et al. 2005). Frequent and energetic CMEs from active stars can also contribute to the stellar angular momentum loss (Drake et al. 2013; Osten \& Wolk 2015; Odert et al. 2017). The interaction of frequent and energetic CMEs with magnetically dominated regions of exoplanetary atmospheres (magnetospheres) can cause generation of electric currents that heat upper atmospheres and cause atmospheric escape and chemical changes (Cohen et al. 2014; 2018; Airapetian et al. 2017a;b; Garcia-Sage et al. 2017; Dong et al. 2017; 2018). The relationship between the eruptive flare and its resulting CME is well understood theoretically (Forbes 2000; Lynch et al. 2016; 2019). Because over $95 \%$ of powerful solar flares are accompanied by CMEs, and the physics of this correlation is understood (as discussed earlier), we will follow other teams to assume that active G-K stars with their frequent and powerful flares could also have correspondingly numerous energetic CMEs. Thus, the frequency of occurrence and energies of flares can characterize the frequency of occurrence and energies of associated CMEs (Gopalswamy et al. 2005; Odert et al. 2017; Lynch et al. 2019). Theoretical models of solar CMEs suggest that they are driven by the evolution and interaction between the low-lying energized and overlying restraining magnetic fields in solar and stellar coronae (Lynch et al. 2008, 2009; 2016; Karpen et al. 2012; Masson et al. 2013). Our team has recently modelled an energetic CME $(7 \times 1033 \mathrm{erg})$ from a young solar-like star, $\kappa^{1}$ Cet, based on the observationally derived k1 Cet magnetogram (Lynch et al. 2019). Magnetic reconnection during the stellar flare creates the twisted flux rope structure of the ejecta and the $\sim 2000 \mathrm{~km} / \mathrm{s}$ CME eruption of magnetic flux forms a strongly magnetized shock.
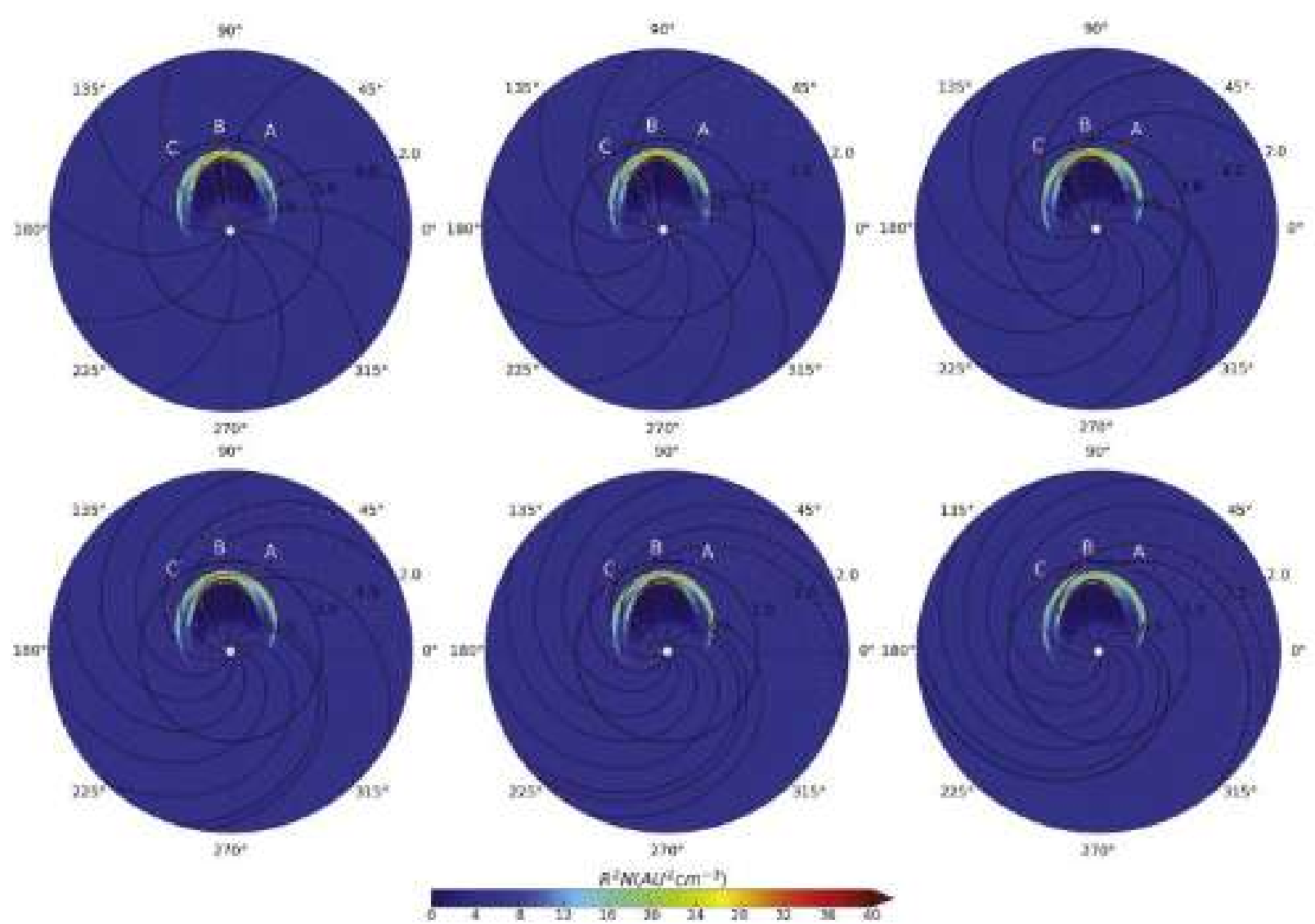

Figure 4. . Configuration for the CME-driven shock under six stellar rotation scenarios at $\mathrm{t}=30.2 \mathrm{hr}$ after CME eruption. The shock is initiated with the nose toward $\mathrm{f}=100^{\circ}$. The top row, from left to right, shows $\Omega=0.5 \Omega_{\odot}$ (case I), $1.0 \Omega_{\odot}$ (case II), and $1.5 \Omega_{\odot}$ (case III), respectively; the bottom row, from left to right, shows $\Omega=2.0 \Omega_{\odot}$ (case IV), $2.5 \Omega_{\odot}$ (case V), and $3.0 \Omega_{\odot}$ (case VI), respectively. The color scheme is for the normalized density nr2. The reference points labeled as A, B, and C are located at longitudes of $70^{\circ}, 100^{\circ}$, and $130^{\circ}$, respectively. 
Strong shocks are the sites of particle acceleration at the shock front via the diffusive shock acceleration (DSA) mechanism (e.g. Zank et al. 2000). Because the shocks are spatially extended and the acceleration often lasts over an extended period, accelerated particles can be observed at multiple locations that are longitudinally well separated, and the time-intensity profile of these events are "gradual" in nature. Our improved Particle Acceleration and Transport in Heliosphere (iPATH) model successfully explains SEP energy flux evolution in time, fluence, $\mathrm{F}$ (the total number of particles per unit area), the energy spectra and their maximum energy. Recent studies suggest that if SEPs with hard energy spectra ( $\mathrm{F} \mathrm{E}^{-\gamma}, \gamma$ i -2) from the young solar-like stars penetrate into the lower layers of exoplanetary atmospheres, they become instrumental in initiating chemical reactions producing complex biomolecules in the atmospheres of early Earth and young exoplanets (Fu et al. 2019; Hu et al. 2021; Airapetian et al. 2016; 2020). Thus, the mechanisms that drive hard energy spectra and high fluence SEPs are of critical importance for understanding atmospheric chemistry of rocky exoplanets around active stars.

Specifically, we studied the role of a CME shock geometry on the fluences and spectra of SEP event cause by a CME with the speed of $1500 \mathrm{~km} / \mathrm{s}$. The shape of the shock for a given CME is determined by the geometry of the global magnetic field of the star, which in turn depends on the stellar rotation rate. We have studied 6 case of stellar rotation periods in the range between 0.5 to 3 times the current solar rotation rate on the fluences and spectra of SEP event cause by a CME with the speed of $2000 \mathrm{~km} / \mathrm{s}$. To evaluate the effect of the CME shock geometry, we kept the stellar magnetic field fixed and equal to the strength of the current Sun. As the star rotates faster, its interplanetary magnetic field (IMF) is described by a tighter Parker spiral presented in Fig. 4.

Our results suggest that the rotation rate alters the shock geometry. The maximum energy gained by a particle at the shock front also depends on the shock geometry. In addition, the observed time-intensity profiles and event-integrated spectra depend on the stellar rotation rate. Our results provide the first insight into the SEP properties in response to various magnetic field geometries and form a framework to study stellar SEPs including their fluence, spectra, and maximum energy from young magnetically active planet hosting stars.

\section{Impact of Stellar Energetic Particles on Atmospheric Chemistry}

Exoplanetary atmospheric chemistry is affected by many internal and external factors including the combined effect of XUV-UV and SEP driven particle precipitation into an exoplanetary atmosphere. The initial atmospheric chemical composition is defined by the interplay between the atmospheric escape and volcanic outgassing of species including $\mathrm{SO} 2, \mathrm{H} 2, \mathrm{CO} 2, \mathrm{H} 2 \mathrm{~S}$, and $\mathrm{H} 2 \mathrm{O}$, among other species, driven by the internal planetary dynamics (see review paper, Airapetian et al. 2020). The interaction of internal and external energy sources such as meteor impacts, lightning, gamma rays, and SEPs can significantly modify atmospheric chemistry and impact the production rate of prebiotic atmospheric chemistry (Airapetian et al. 2016; and review in Airapetian et al. 2020; Patel et al. 2015). The atmospheric synthesis of molecules of astrobiological interest is influenced by the amount of energy available to drive such chemistry and by the altitude at which that energy is delivered. The main source of external energy, stellar XUV-UV $(1-300 \mathrm{~nm})$ radiation, will be augmented by reactions induced by other nonthermal processes: ionization, dissociation, and excitation of atomic/molecular species by energetic particles. Of critical importance to the origin of life are processes that dissociate N2 and produce 'fixed nitrogen' that can be used biologically. Stellar EUV radiation can do so, but that process typically happens high up in a planet's atmosphere (see, e.g., Zahnle, 1986; Airapetian et al. 2020). SEPs are much more penetrating and can dissociate N2 all the way down into the exoplanetary troposphere (Airapetian et al., 2016; 2020). Galactic Cosmic Rays and lightning can also cause N2 dissociation at low altitudes, but these sources have been studied previously (Chameides and Walker, 1981; Wong et al., 2017) and will be only used as a comparison against the solar induced production in our modeling.

Given a range of computed atmospheric compositions (see Table 1), we can calculate the steady-state concentrations of species that might have played a role in prebiotic chemistry. These include formaldehyde, H2CO (Pinto et al., 1980) and HCN (Zahnle, 1986), along with various oxides of nitrogen. The main species that we expect to produce initially from SEP bombardment are nitric oxide, NO, and carbon monoxide, $\mathrm{CO}$, as it follows from the reaction

$$
\frac{1}{2} N_{2}+C O_{2}->N O+C O[R 1]
$$


As mentioned earlier, energetic particles break the $\mathrm{N} 2$ triple bond, producing $\mathrm{N}$ atoms which can then strip oxygen from $\mathrm{CO} 2$ to produce $\mathrm{NO}$ and $\mathrm{CO}$. $\mathrm{CO}$ itself is interesting in a prebiotic context, as it is a high-energy compound that could have accumulated to substantial concentrations in the early planetary atmosphere (Kasting, 1990). It could have provided a free energy source for early metabolism by way of the water-gas shift reaction: $\mathrm{CO}+\mathrm{H}_{2} \mathrm{O}+\mathrm{CO}_{2}+\mathrm{H}_{2}$ (Kasting, 2014). $\mathrm{CO}$ also plays a role in some models of the origin of life (Huber and Wachtershauser, 1997, 1998). These authors envisioned an origin of life in hydrothermal vent systems; however, the free energy from the water-gas shift reaction should also have been available in surface environments. Other sources of energy could also have driven reaction R1, breaking N2 bonds. Previously recognized processes for doing this include lightning (Yung \& McElroy 1979; Kasting Walker 1981; Navarro-Gonzalez et al. 1998; Wong et al. 2017), bolide impacts (McKay et al. 1988), and hot volcanic vents (Mather et al. 2004). But model calculations based on Airapetian $(2016,2020)$ suggest that hard spectra SEP bombardment may have been more effective than any of these other processes in producing NO.

The NO produced by reaction R1 could have played an important role in prebiotic chemistry. In the atmosphere, $\mathrm{NO}$ would have been converted to $\mathrm{HNO}$ and, to a lesser extent, $\mathrm{HNO}_{2}$ and $\mathrm{HNO}_{3}$ (Kasting Walker 1981; Wong et al. 2017). These hydrated forms of NO are all highly soluble and would have rained out into the early ocean. Once there, HNO would then have undergone dissociation, homologation, and decay reactions, ultimately yielding nitrite $\left(\mathrm{NO}_{2}\right)$, nitrate $\left(\mathrm{NO}_{3}\right)$, and gaseous $\mathrm{N}_{2} \mathrm{O}$ (Mancinelli \& McKay, 1988; Summers \& Khare, 2007; Hu and Diaz, 2019). The N2O would have escaped to the atmosphere, where most of it should have been photodissociated but where, on exoplanets, it could also be confused as a biosignature. Meanwhile, nitrate and nitrite are implicated in a wide range of prebiotic chemistries (Laneuville et al. 2018; Ranjan et al. 2019; Mariani et al. 2018; Becker et al. 2019). Recent work suggests that their concentrations would have been low in the early oceans because of reaction with dissolved ferrous iron, but they could have been more abundant in prebiotic lakes (Ranjan et al., 2019). These predictions are broadly consistent with recently derived isotopic constraints (Homann et al. 2018). NO itself could have participated in prebiotic chemistry by serving as a strong electron sink (Ducluzeau et al., 2009).

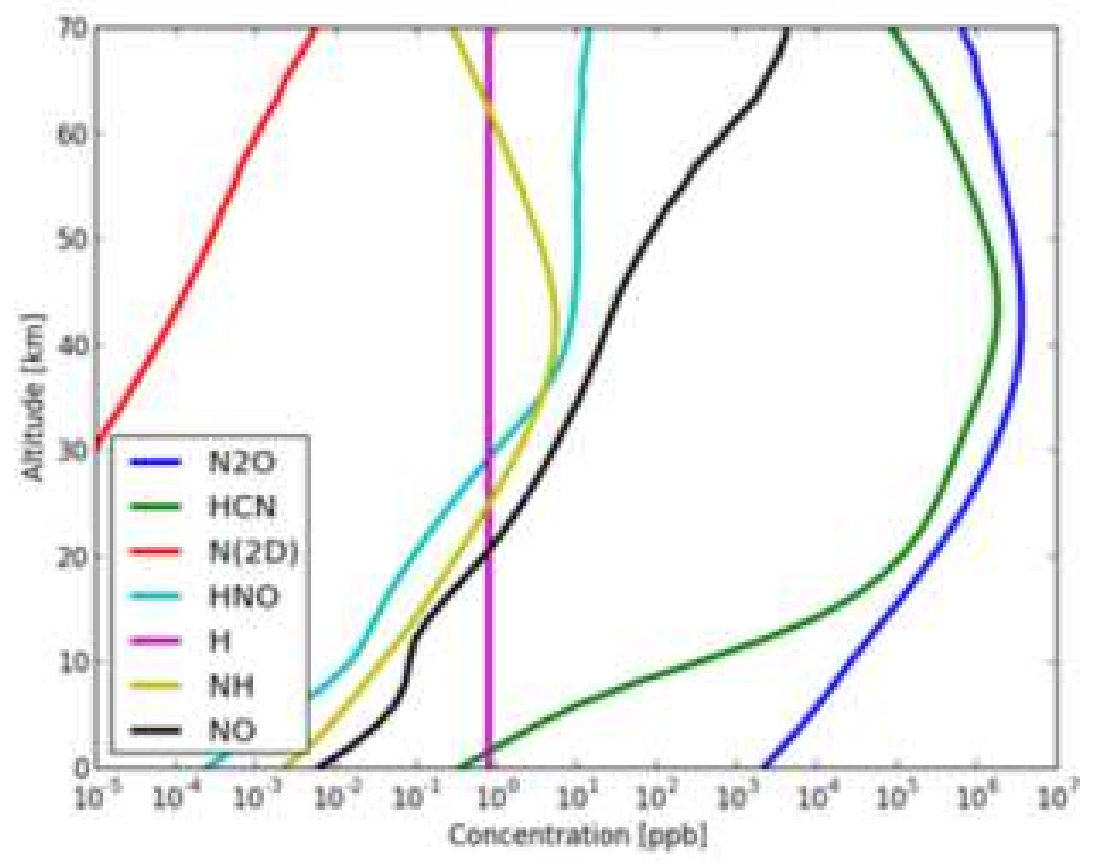

Figure 5. Vertical profiles of biologically important molecules produced by an SEP irradiation of a $\mathrm{N}_{2}-\mathrm{CO}_{2}$ rich atmosphere.

Another key molecule of interest is HCN, which serves as a precursor for many different biomolecules and prebiotic compounds. The main source of HCN on the early Earth has been considered to be recombination of $\mathrm{N} 2+$ in the ionosphere, producing $\mathrm{N}$ atoms. These flow down into the stratosphere where they react with the byproducts of methane photolysis to produce $\mathrm{HCN}$, e.g.: $\mathrm{CH} 3+\mathrm{N}+\mathrm{HCN}+\mathrm{H}_{2} ; 3 \mathrm{CH}_{2}+\mathrm{N}+\mathrm{HCN}$ $+\mathrm{H}$ (Zahnle, 1986; Tian et al., 2011). HCN can also be produced in lightning discharges but only if the 
atmospheric C:O ratio is $>1$ (Chameides and Walker, 1981; Rimmer and Rugheimer, 2019). In practice, this requires $\mathrm{CH} 4: \mathrm{CO} 2 i, 1$, which is not thought to be true for realistic prebiotic atmospheres, for which researchers struggle to justify CH4 concentrations i10-100 ppmv (Kasting and Catling, 2003). Impacts are a more likely source of HCN because of the reduced nature of the gases within the impact plume (Ferus et al., 2017; Ritson et al., 2018). Once formed, HCN would have rained out of the atmosphere and entered the oceans, where it would have participated in numerous reactions. The hydrolysis rate of HCN as a function of $\mathrm{pH}$ and temperature has been carefully measured (Miyakawa et al. 2002).

Our updated model accounts for a number of factors including eddy diffusion and convection effects, concentration of hazes or Rayleigh scattering of solar EUV radiation in the atmosphere that significantly reduces photo-destruction of $\mathrm{N}_{2} \mathrm{O}$, and therefore increases its production. Typical fluence of SEP events from the young Sun and magnetically active G-M dwarfs could have been a factor of 10-100 greater than that conservatively assumed in our model.

Laboratory experiments report the production of nitrogen oxides, including N2O, when N2-CO2 mixtures that simulate primitive early Earth atmosphere were exposed to lightning or coronae discharges (NnaMvondo et al. 2005). Enhanced production of $\mathrm{N}_{2} \mathrm{O}$ in lighting experiments were explained by energetic electrons accelerated in the discharge and UV emission. The production of $\mathrm{CH}, \mathrm{NH}$ and $\mathrm{NO}$ sets stage for the formation of $\mathrm{HCN}=$, and other N-containing species in the lower parts of the atmosphere, which may subsequently rain out into surface reservoirs and engage in higher order chemistry producing more complex organics. The calculated production rate of $\mathrm{HCN}$ in the low atmosphere is driven by three major reactions: $\mathrm{CH}+\mathrm{N}+\mathrm{HCN}, \mathrm{CH}_{3}+\mathrm{N}+\mathrm{HCN}+\mathrm{H}_{2}$ and $\mathrm{CH}_{2}+\mathrm{N}+\mathrm{HCN}+\mathrm{H}$. This implies the production rate of $\mathrm{HCN}$ about $10^{3} \mathrm{Tg} / \mathrm{yr}$. Further HCN polymerization is known to produce various amino acids, the building blocks of proteins (Miyakawa et al. 2002; Coustenis et al. 2007), and is a starting material in the synthesis of purine and pyrimidine nucleobases in addition to sugars via a Kiliani-Fischer mechanism. Production of other types of soluble $\mathrm{N}$-containing species (such as $\mathrm{NH}_{3}$ and $\mathrm{NOx}$ ) by SEPs may have provided a massive dose of nitrogen "fertilizer" to early surface biology on terrestrial planets. Our new concept of SEP mediated chemistry challenges the current view of the early Earth atmosphere and predicts new avenues for prebiotic chemistry of young exoplanets around active G-K stars. This model suggests the crucial role of N2O in warming the early Earth and young rocky exoplanets providing new pathway for formation of HCN and other nitrogen bearing molecules conducive to prebiotic chemistry as well as the resolution of the FYS paradox (Feulner 2012; Airapetian et al. 2020).

\section{Conclusion}

Here we described a novel approach in assessing the drivers and products of atmospheric chemistry of rocky exoplanets exposed to high XUV and SEP fluxes associated with flares from their active planet hosts. We applied an MHD codem AWSoM, to model the quiescent solar corona and the wind from young solarlike stars. This plasma and magnetic background was then used to study the properties of stellar energetic particles by using a proven heliophysics tool, iPATH, for a wide range of stellar environments, an unexplored source of chemical changes in exoplanetary atmospheres. This study will also have a major impact on our understanding of the rise of prebiotic atmospheric chemistry on Earth and rocky exoplanets, associated effects on climate, chemical synthesis and habitability of rocky planets, by placing them in a more complete context with respect to the history of magnetic activity of their host stars. The future work will study how high-energy events in young and active G-K stars may affect evolution of prebiotic and primitive biospheric chemistry and atmospheric warming mechanisms of rocky exoplanets. 


\section{References}

Hu, J., Airapetian, V. S., Li, G., Zank, G., Jin, M. 2021, Extreme Energetic Particle Events by Superflare Associated CMEs from Solar-like Stars, Science Advances.

Airapetian, V. S., Jin, M., Lüftinger, T., Boro Saikia, S., Kochukhov, O., Gudel, M., B. Van DerHolst 2021, One Year in the Life of Young Suns, Astrophys. J., 916, 96.

Airapetian, V. S., Barnes, R., Cohen, O. et al. 2020, Int. J. of Astrobio. 19(2), 136.

Airapetian, V. S. 2017, in Extreme Events in Geospace: Origins, Predictability, and Consequences, Chapter 25. p. 611-632.

Airapetian, V. S., Glocer, A., Khazanov, G. V. et al. 2017a, Astrophys.-J. Lett., 836, L3.

Airapetian, V. S., Jackman, C. H., Mlynczak, M., Danchi, W., Hunt, L. 2017b, Atmospheric Beacons of Life from Exoplanets Around G and K Stars, Nature Scientific Reports, 7, 14141.

Airapetian, V. S., Glocer, A., Gronoff, G., E. Hébrard, E., Danchi, W. 2016, Nature Geoscience, 9, 452.

Airapetian, V. \& Usmanov, A. 2016, Astrophys. J. Lett., 817, L24.

Alvarado-Gomez, J. D., Hussain, G. A. J., Cohen, O., et al. 2016, Astron. Astrophys., 588, A28

Barth, P., Helling, Ch., Stüeken, E. E. et al. 2021, Mon. Not. R. Astron. Soc., 502, 6201.

Beard, D. B. 1960, J. Geophys. Res., 65(11), 3559, 10.1029/JZ065i011p03559.

Benedict, G. F., McArthur, B. E., Gatewood, G., et al. 2006, Astron. J, 132, 2206

Boro Saikia, S., Jin, M., Johnstone, C. P. et al. 2020. Astron. Astrophys., 635, A178.

Boro Saikia, S., Jeffers, S. V., Petit, P., et al. 2015, Astron. Astrophys., 573, A17.

Cohen, O., Drake, J. J., Glocer, A. et al. 2014, Astrophys. J., 790, article id. 57, 13.

Cranmer, S. R. and Saar, S. H. 2011, Astrophys. J., 741, 54.

Do Nascimento, Jr. J-D, Vidotto, A. A., Petit, P. et al. 2016, Astrophys. J. Lett., 820, L15.

Dong, C. F., Jin, M., Lingam, M., Airapetian, V. S., Ma, Y. J., van der Holst, B. 2018, Proc. Nat. Acad. Sci., $115,260$.

Fu, S., Jiang, J., Airapetian, V., Hu, J., Li, G., and Zank, G., 2019, Astrophys. J. Lett., 878, L36.

Garcia-Sage, K., Glocer, A., Drake, J. J. et al. 2017, Astrophys. J. Lett., 844, L13.

Güdel, M. 2020, Space Science Reviews, 216, article id.143.

Johnstone, C., Khodanchenko, M. and Lüftinger, T. et al. 2019, Astron. Astrophys., 624, L10.

Kochukhov, O., Hackman, T., Lehtinen, J. J. 2020, Astron. Astrophys., 635, 142.

Lynch, B. J., Airapetian, V. S., DeVore, C. R. et al. 2019, Astrophys. J., 880, 97-109.

Maehara H., Shibayama T., Notsu S. et al 2012, Nature 485, 478.

Parker, E. N. 1958, Astrophys. J., 128, 664.

Patel, B. H., Percivalle, C., Ritson, D. J., Duffy, C. D., \& Sutherland, J. D. 2015, Nature Chemistry, 7, 301.

Ribas, I., Guinan, E. F., Gudel, M. et al. 2005, Astrophys. J., 622, 680.

Rosen, L., Kochukhov, O., Hackman, T.,\& Lehtinen, J. 2016, Astron. Astrophys,, 593, A35.

Sanz-Forcada, J., Micela, G., Ribas, I. et al. 2011, Astron. Astrophys., 532, A6. 
See, V., Matt, S. P., Folsom, C. P. et al. 2019, Astrophys. J., 876, 118.

Vidotto, A. 2021, Living Reviews in Solar Physics, in press, 10.1007/s41116-021-00029- w

Vidotto, A. \& Cleary, A. 2020, Mon. Not. R. Astron. Soc., 494, 2417.

Waite I. A., Marsden, S. C., Carter, B. D. et al., 2017, Mon. Not. R. Astron. Soc., 465, 2076. 